\title{
Cubic Function-Based Bayesian Dynamic Linear Prediction Approach of Bridge Extreme Stress
}

\author{
Yuefei Liu, ${ }^{1,2}$ Qingkai Xiao,, ${ }^{1,2}$ and Xueping Fan ${ }^{1,2}$ \\ ${ }^{1}$ Key Laboratory of Mechanics on Disaster and Environment in Western China, Ministry of Education of China, \\ Lanzhou University, Lanzhou, China \\ ${ }^{2}$ School of Civil Engineering and Mechanics, Lanzhou University, Lanzhou 730000, China \\ Correspondence should be addressed to Xueping Fan; fanxp@lzu.edu.cn
}

Received 17 June 2017; Accepted 10 September 2017; Published 24 October 2017

Academic Editor: Francesco Ruffino

Copyright (C) 2017 Yuefei Liu et al. This is an open access article distributed under the Creative Commons Attribution License, which permits unrestricted use, distribution, and reproduction in any medium, provided the original work is properly cited.

\begin{abstract}
In structural health monitoring (SHM) field, the structural stress prediction and assessment are the research bottleneck. To reasonably and dynamically predict structural extreme stress based on the time-variant monitored data, the objectives of this paper are to present (a) cubic function-based Bayesian dynamic linear models (BDLM) about monitored extreme stress, (b) choosing method of optimum probability distribution functions about initial stress state, (c) monitoring mechanism of the optimum BDLM, and (d) an effective way of taking advantage of BDLM to incorporate the time-variant monitored data into structural extreme stress prediction. The monitored data of an existing bridge is adopted to illustrate the feasibility and application of the proposed models and procedures.
\end{abstract}

\section{Introduction}

Nowadays, most of the service bridges are close or above their planned lifetime at home and abroad. The bridge stress can reflect the structural basic status. Therefore, it is crucial to predict the structural dynamic stress for the safety and serviceability assessment of critical infrastructure components for bridge systems. Therefore, how to build the dynamic prediction model of structural stress becomes very important.

For bridges, SHM is a very useful tool which can provide the structural basic status, including strain, stress, and deflection of specified structural components or structures. How to properly handle with the continuously provided monitored data is one of the main difficulties in the SHM field. Up to now, a sound number of studies about the data handling are mainly focused on the modal parameter identification, structural damage detection technology, data modeling, and so on [1-4]. For bridge performance prediction and assessment based on SHM data, some achievements have been obtained, for instance, extreme stress prediction of bridge based on BDLM and monitored data [5-7], Bayesian prediction of structural bearing capacity of aging bridges based on dynamic linear model [8], and structural performance prediction using monitored extreme data [9]. These above dynamic linear prediction models are all built with linear functions. Compared with the dynamic linear models built with quadratic function [10], the prediction precision of linear models built with linear functions is lower. Therefore, based on the dynamic SHM data of bridges, the research on building more reasonable dynamic linear models for predicting the structural extreme stress should be further studied.

In this paper, considering the uncertainty of structural dynamic monitored data and the diversity of initial stress state probability distributions, the optimum Bayesian dynamic linear prediction method about monitored extreme stress including nonlinear effects is given with cubic function, and the detailed contents are described in detail as follows.

Firstly, build cubic function of monitored extreme stress. Secondly, Bayesian dynamic linear model is established based on the built cubic function.

Thirdly, considering the diversity of probability distribution functions (PDF) about initial state information, the optimum PDF is chosen with Kullback-Leibler (K-L) information 


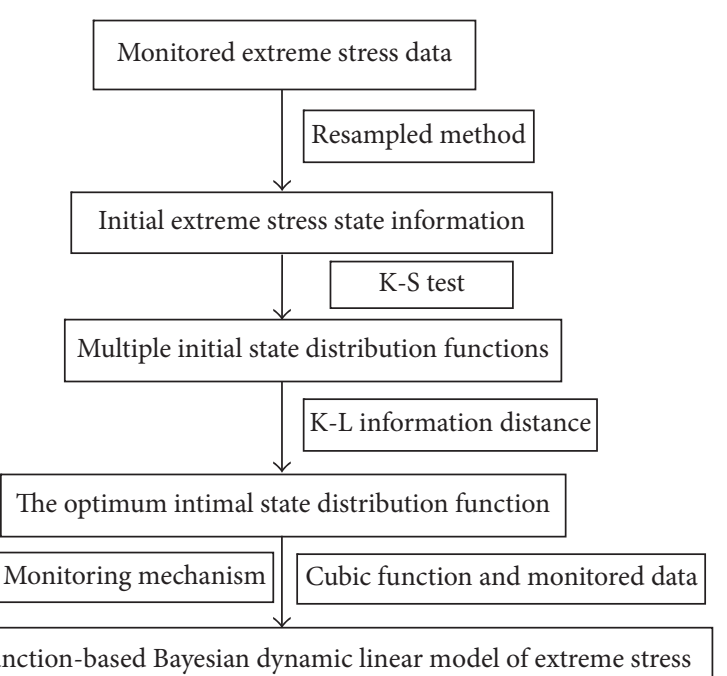

Cubic function-based Bayesian dynamic linear model of extreme stress

FIGURE 1: The building processes of the optimum Bayesian dynamic linear model.

distance. And then the optimum BDLM and the corresponding model monitoring mechanism are, respectively, given.

Finally, an actual engineering is provided to illustrate the feasibility and application of the proposed model and method in this paper.

Cubic function-based Bayesian dynamic linear prediction approach of bridge extreme stress is shown in Figure 1.

\section{BDLM}

BDLM is a predicting approach based on a philosophy of information updating [11-14] which defines a dynamic model system of time series processes that can incorporate all useful monitored information into the model to update the prediction. It comprises a state equation, a monitored equation, and the a priori information. The state equation, which shows changes of the system with time and reflects inner dynamic changes of the system and random disturbances, is linear. The monitored equation, which expresses the relationship between the monitoring data and the current state parameters of the system, is also linear.

In this paper, the cubic function is adopted to build the BDLM, the prediction precision of which is better than the BDLM built based on linear function model and quadratic function model about the SHM data. The cubic functionbased BDLM is described in detail as follows.

2.1. Assumptions of the BDLM. BDLM is presented as a special case of a general state-space model, being linear and Gaussian. So the BDLM satisfies the assumptions of a statespace model, while the basic assumptions $[11,12]$ of a statespace model are as follows:

(a) State variables, monitored errors, and state errors all follow normal/Gaussian distributions.

(b) $\left(\theta_{t}\right)$ is a Markov chain $[11,12]$. Namely, $\pi\left(\theta_{t+1} \mid \mathbf{D}_{t}\right)=$ $\pi\left(\theta_{t+1} \mid \theta_{0: t}, y_{1: t}\right)=\pi\left(\theta_{t+1} \mid \theta_{t}\right)$, where $\pi(\cdot)$ is probability density function and $\theta_{t}$ is the state variable at time $t$. (c) Conditionally on $\theta_{t}, y_{t}, t=1,2,3, \ldots, T$ are independent of each other and $y_{t}$ depends on $\theta_{t}$ only, where $y_{t}$ is the monitored variable at time $t$.

\subsection{Cubic Function and Dynamic Linear Model of Monitored} Extreme Stress. For the long-term health monitoring extreme stress data, the fitted cubic function $h(t)$ shown in (1) can be more approximately and reasonably adopted than linear function and quadratic function to build the state equation, namely,

$$
h(t)=a t^{3}+b t^{2}+c t+d,
$$

where $h(t)$ is the trend data of the monitored information. $a$, $b, c$, and $d$ are all regression coefficients. $t=$ time, the unit of which is day.

$$
\begin{aligned}
\theta_{t-2} & =h(t-2)=a(t-2)^{3}+b(t-2)^{2}+c(t-2)+d, \\
\theta_{t-1} & =h(t-1)=a(t-1)^{3}+b(t-1)^{2}+c(t-1)+d, \\
\theta_{t} & =h(t)=a t^{3}+b t^{2}+c t+d, \\
\theta_{t+1} & =h(t+1)=a(t+1)^{3}+b(t+1)^{2}+c(t+1)+d,
\end{aligned}
$$

where $\theta_{t-2}, \theta_{t-1}, \theta_{t}$, and $\theta_{t+1}$ are, respectively, the state data at times $t-2, t-1, t$, and $t+1$, namely, the trends of the monitoring data.

$$
\begin{aligned}
\Delta_{t+1} & =\theta_{t+1}-\theta_{t}=3 a t^{2}+(3 a+2 b) t+a+b+c, \\
\Delta_{t} & =\theta_{t}-\theta_{t-1}=3 a t^{2}+(2 b-3 a) t+a+c-b, \\
\Delta_{t-1} & =\theta_{t-1}-\theta_{t-2}=3 a t^{2}+(2 b-9 a) t+7 a+c-3 b,
\end{aligned}
$$

where $\Delta_{t+1}$ is the change of $\theta$ from time $t$ to time $t+1, \Delta_{t}$ is the change of $\theta$ from time $t-1$ to time $t$, and $\Delta_{t-1}$ is the change of $\theta$ from time $t-2$ to time $t-1$.

$$
\begin{aligned}
\xi_{t+1} & =\Delta_{t+1}-\Delta_{t}=6 a t+2 b, \\
\xi_{t} & =\Delta_{t}-\Delta_{t-1}=6 a t-6 a+2 b, \\
\xi_{t+1} & =\xi_{t}+6 a,
\end{aligned}
$$

where $\xi_{t+1}$ is the change of $\Delta$ from time $t$ to time $t+1$ and $\xi_{t}$ is the change of $\Delta$ from time $t-1$ to time $t$.

Further, with (1)-(4) and considering the uncertainty and the randomness of the monitored stress data, the BDLM is built as follows:

Monitored equation is

$$
y_{t+1}=\theta_{t+1}+v_{t+1}, \quad v_{t+1} \sim N\left[0, V_{t+1}\right] .
$$

State equation is

$$
\begin{aligned}
& \theta_{t+1}=\theta_{t}+\Delta_{t+1}+\omega_{t+1,1}, \\
& \Delta_{t+1}=\Delta_{t}+\xi_{t+1}+\omega_{t+1,2}, \quad \xi_{t+1}=\left(\frac{r_{i+1}}{r_{i}}\right)\left(\xi_{t}+6 a\right)+\omega_{t+1,3}, \\
& \boldsymbol{\omega}_{t+1}=\left\{\begin{array}{l}
\omega_{t+1,1} \\
\omega_{t+1,2} \\
\omega_{t+1,3}
\end{array}\right\} \sim N\left[0, \mathbf{W}_{t+1}\right], \quad t=1,2, \ldots, T .
\end{aligned}
$$




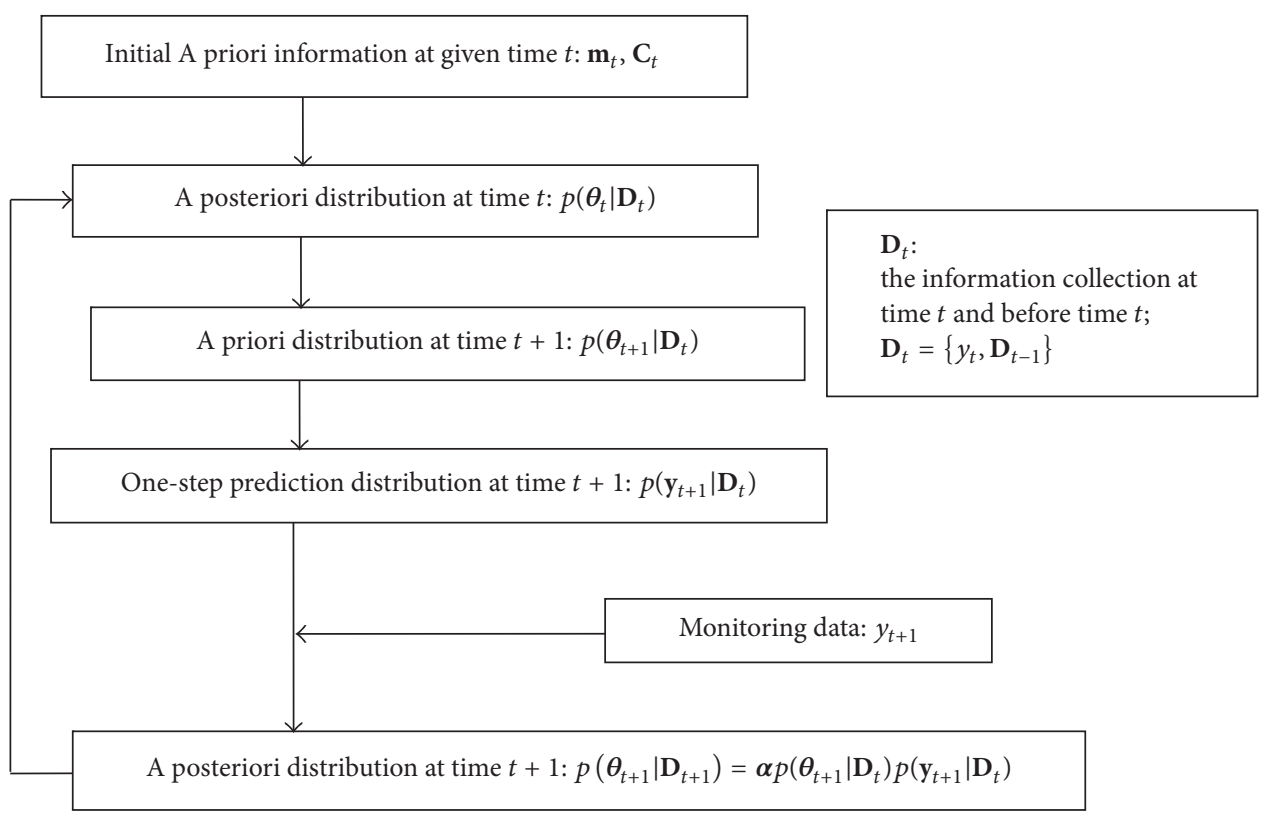

FIGURE 2: The probability recursive processes of BDLM.

For short,

$$
\begin{aligned}
\left(\begin{array}{c}
\theta_{t+1} \\
\Delta_{t+1} \\
\xi_{t+1}
\end{array}\right)=\mathbf{G}_{t+1}\left(\begin{array}{c}
\theta_{t} \\
\Delta_{t} \\
\xi_{t}+6 a
\end{array}\right)+\boldsymbol{\omega}_{t+1}, & \\
\mathbf{G}_{t+1} & =\left(\begin{array}{ccc}
1 & 1 & \frac{r_{i+1}}{r_{i}} \\
0 & 1 & \frac{r_{i+1}}{r_{i}} \\
0 & 0 & \frac{r_{i+1}}{r_{i}}
\end{array}\right) .
\end{aligned}
$$

Initial state information is

$$
\left(\left[\begin{array}{c}
\theta_{t} \\
\Delta_{t} \\
\xi_{t}+6 a
\end{array}\right] \mid \mathbf{D}_{t}\right) \sim N\left[\mathbf{m}_{t}, \mathbf{C}_{t}\right]
$$

where $y_{t+1}$ is the monitored extreme stress data at time $t+$ 1. $\theta_{t+1}$ is the state at time $t+1$, namely, the trend of the monitoring data at time $t+1 . \Delta_{t+1}$ is the change of $\theta$ from time $t$ to time $t+1 . \xi_{t+1}$ is the change of $\Delta$ from time $t$ to time $t+1$. $v_{t+1}$ is the monitored error. $\omega_{t+1}$ is state error vector. $\mathbf{D}_{t}$ is the information collection including the data at time $t$ and before time $t$, namely, $\mathbf{D}_{t}=\left\{y_{t}, \mathbf{D}_{t-1}\right\}, \mathbf{D}_{t-1}$ is the data collection at time $t-1$ and before time $t-1$, including $\mathbf{m}_{t-1}$ (mean value matrix), $\mathbf{C}_{t-1}$ (variance matrix), and so on. Moreover, suppose that $v_{t+1}$ and $\omega_{t+1}$ are mutually independent and independent of $\theta_{t+1} \cdot r_{i+1}=t_{i+1}-t_{i}$ is the sampling interval,

$$
\mathbf{m}_{t}=\left[\begin{array}{c}
m_{\theta_{t}} \\
m_{\Delta_{t}} \\
m_{\xi_{t}}
\end{array}\right]
$$

$$
\begin{aligned}
\mathbf{C}_{t} & =\left[\begin{array}{ccc}
C_{\theta_{t}} & C_{(\theta, \Delta)_{t}} & C_{(\theta, \xi)_{t}} \\
C_{(\theta, \Delta)_{t}} & C_{\Delta_{t}} & C_{(\Delta, \xi)_{t}} \\
C_{(\xi, \theta)_{t}} & C_{(\xi, \Delta)_{t}} & C_{\xi_{t}}
\end{array}\right], \\
\mathbf{W}_{t+1} & =\left[\begin{array}{lll}
W_{t+1,1} & W_{t+1,4} & W_{t+1,5} \\
W_{t+1,4} & W_{t+1,2} & W_{t+1,6} \\
W_{t+1,5} & W_{t+1,6} & W_{t+1,3}
\end{array}\right] .
\end{aligned}
$$

For BDLM, $v_{t+1}$ and $\omega_{t+1}$ are, respectively, monitored errors and state errors. It is assumed that error sequences $v_{t+1}$ and $\omega_{t+1}$ are internally independent, mutually independent, and independent of $\left(\theta_{t} \mid \mathbf{D}_{t}\right)$.

With (5)-(8), the updating relationship between monitored data and state parameters can be obtained as

$$
\begin{aligned}
\left(y_{t+1} \mid \theta_{t+1}\right) & \sim N\left[\theta_{t+1}, V_{t+1}\right], \\
\left(\theta_{t+1} \mid \theta_{t}\right) & \sim N\left[m_{\theta_{t}}+m_{\Delta_{t}}+\frac{r_{i+1} m_{\xi_{t}}}{r_{i}}, W_{t+1,1}\right] .
\end{aligned}
$$

It is known from (10) that the modeling processes of BDLM can be divided into two key steps, which are shown in Figure 2. The first step is to obtain a priori probability distribution function (PDF) of $\theta_{t+1}$ based on the state equation and a posteriori PDF of $\theta_{t}$. The second step is to get a posteriori $\operatorname{PDF}$ of $\theta_{t+1}$ based on a priori PDF of $\theta_{t+1}$ and monitored data $y_{t+1}$.

\subsection{Determination of the Main Probability Parameters about} $B D L M$. For the BDLM, the probability parameters need to be solved include $V_{t+1}, \mathbf{W}_{t+1}, \mathbf{m}_{t}$, and $\mathbf{C}_{t}$. The determining methods about these parameters are described as follows. 
In this paper, $V_{t+1}$ is estimated with the variance of differences between fitted trend data $\theta_{t+1}$ and monitored extreme stress data $y_{t+1}$. According to $[11,12], W_{t+1}$ can be solved with

$$
W_{t+1}=\left(\frac{1-\delta}{\delta}\right) G_{t+1} C_{t} G_{t+1}^{\prime}
$$

where $G_{t+1}^{\prime}$ is the transpose of $G_{t+1} . \delta$ is the discount factor, which is usually $0.95-0.98$ given by engineering experience of the authors.

$m_{t}$ is the mean value of state variable $\left(\theta_{t}, \Delta_{t}, \xi_{t}\right)$ at time $t$. $C_{t}$ is the variance of state variable $\left(\theta_{t}, \Delta_{t}, \xi_{t}\right)$ at time $t$. They can be both estimated with the resampled monitored data $\theta$, the change $\Delta$ of $\theta$, and the change $\xi$ of $\Delta$ at time $t$ and before time $t$.

Considering the diversity of PDF about the state variable including $\left(\theta_{t+1}, \Delta_{t+1}, \xi_{t+1}\right)$ which are obtained and resampled based on the mass SHM data, the K-L information distance is adopted to determine the optimum PDF, namely,

$$
\begin{aligned}
D_{\mathrm{KL}, i}^{2}\left(\theta_{t+1}\right)=\int p_{R T, i}\left(\theta_{t+1}\right) \ln \frac{p_{R T, i}\left(\theta_{t+1}\right)}{p_{R}\left(\theta_{t+1}\right)} d \theta_{t+1}, & \\
& i=1,2, \ldots,
\end{aligned}
$$

where $D_{\mathrm{KL}, i}^{2}\left(\theta_{t+1}\right)$ is the K-L information distance, $p_{R T, i}\left(\theta_{t+1}\right)$ is the $i$ th common PDF about $\theta_{t+1}$, and $p_{R}\left(\theta_{t+1}\right)$ is the theoretical PDF about $\theta_{t+1}$ obtained with kernel density estimation method. If $D_{\mathrm{KL}, i}^{2}\left(\theta_{t+1}\right)$ is smaller, then the corresponding $p_{R T, i}\left(\theta_{t+1}\right)$ is more reasonable. $p_{R T, i}\left(\Delta_{t+1}\right)$ and $p_{R T, i}\left(\xi_{t+1}\right)$ can be also obtained with (12).

The extension of common PDF is better than theoretical PDF, and more reasonably used for BDLM, so the K-L information distance is adopted to determine the optimum common PDF about the state variable.

If the initial state variable follows the other common PDF except Gaussian PDF, then the distribution can be approximately transferred into mixed Gaussian PDF. Take state variable $\theta_{t+1}$ for the example, namely, the following.

(1) With kernel density estimation method, the actual PDF $G\left(\theta_{t+1}\right)$ about the initial state data is approximately $p_{R}\left(\theta_{t+1}\right)$, namely,

$$
G\left(\theta_{t+1}\right) \approx p_{R}\left(\theta_{t+1}\right)
$$

(2) Since any set of data can be fitted by a few normal distributions, namely.

$$
G\left(\theta_{t+1}\right) \approx \sum_{k=1}^{n} p_{k} \Phi\left(\frac{\theta_{t+1}-\mu_{k}}{\sigma_{k}}\right)
$$

where $\sum_{k=1}^{n} p_{k}=1$ and $p_{k} \geq 0, \Phi(\cdot)$ denotes the cumulative probability distribution functions of standard normal distribution.
(3) The weights and distribution parameters of the fitted normal distributions can be obtained with the least residual error quadratic sum method OLS, namely,

OLS

$$
=\sqrt{\frac{1}{n} \sum_{j=1}^{m}\left(g\left(\theta_{t+1, j}\right)-\left(\sum_{i=1}^{n} p_{i} \Phi\left(\frac{\theta_{t+1, j}-\mu_{i}}{\sigma_{i}}\right)\right)\right)^{2}},
$$

where $m$ is the total number of state data and $p_{i}$ is the weight. The values of unknown parameters for the fitted distributions can be possessed by the optimization computation with the rule of OLS. Furthermore, the optimized parameters must be determined to make sure that the value of OLS is the minimum.

With (13)-(15), the common Gaussian PDF can be reached:

$$
p_{R T}\left(\theta_{t+1}\right) \approx \sum_{i=1}^{n} p_{i} \Phi\left(\frac{\theta_{t+1}-\mu_{i}}{\sigma_{i}}\right) .
$$

The transferred common Gaussian $\operatorname{PDF}\left(p_{R T}\left(\Delta_{t+1}\right)\right.$, $\left.p_{R T}\left(\xi_{t+1}\right)\right)$ about the state variables $\left(\Delta_{t+1}, \xi_{t+1}\right)$ can also be approximately obtained with (13)-(16).

2.4. Probability Recursion of BDLM. BDLM are applicable to the prediction of the future state parameters, which can be recursive and updated like well-known Kalman filter [11, 12]. The recursively updating processes [11-14] are as follows.

(1) A Posteriori Distribution at Time t. For the mean $\mathbf{m}_{t}$ and the variance matrix $\mathbf{C}_{t}$, there is

$$
\left(\boldsymbol{\mu}_{t} \mid \mathbf{D}_{t}\right)=\left(\left[\begin{array}{c}
\boldsymbol{\theta}_{t} \\
\Delta_{t} \\
\boldsymbol{\xi}_{t}+6 \mathbf{a}
\end{array}\right] \mid \mathbf{D}_{t}\right) \sim \mathbf{N}\left[\mathbf{m}_{t}, \mathbf{C}_{t}\right]
$$

(2) A Priori Distribution at Time $t+1$

$$
\left(\boldsymbol{\mu}_{t+1} \mathbf{D}_{t}\right) \sim N\left[\mathbf{a}_{t+1}, \mathbf{R}_{t+1}\right],
$$

where, $\mathbf{a}_{t+1}=\mathbf{G}_{t+1} \mathbf{m}_{t}, \mathbf{R}_{t+1}=\mathbf{G}_{t+1} \mathbf{C}_{t} \mathbf{G}_{t+1}^{\prime}+\mathbf{W}_{t+1}=$ $\left[\begin{array}{lll}R_{t+1,1} & R_{t+1,4} & R_{t+1,5} \\ R_{t+1,4} & R_{t+1,2} & R_{t+1,6}\end{array}\right]$

$\left[\begin{array}{lll}R_{t+1,4} & R_{t+1,2} & R_{t+1,6} \\ R_{t+1,5} & R_{t+1,6} & R_{t+1,3}\end{array}\right]$

(3) One-Step Prediction Distribution at Time $t+1$

$$
\left(y_{t+1} \mid \mathbf{D}_{t}\right) \sim N\left[f_{t+1}, Q_{t+1}\right],
$$

where $f_{t+1}=E\left(y_{t+1} \mid y_{i, 1: t}\right)=m_{\theta_{t}}+m_{\Delta_{t}}+\left(r_{i+1} / r_{i}\right) m_{\xi_{t}}, Q_{t+1}=$ $\operatorname{var}\left(y_{t+1} \mid y_{1: t}\right)=R_{t+1,1}+V_{t+1}, u_{t+1}=1 / Q_{t+1}$ is the prediction precision at time $t+1$.

According to the definition of highest posterior density (HPD) region [11], the predicted interval of the monitored data with a $95 \%$ confidential interval at time $t+1$ is

$$
\left[f_{t+1}-1.645 \sqrt{Q_{t+1}}, f_{t+1}+1.645 \sqrt{Q_{t+1}}\right]
$$


where $f_{t+1}-1.645 \sqrt{Q_{t+1}}$ is the predicted lower limit value, $f_{i, t+1}+1.645 \sqrt{Q_{i, t+1}}$ is the predicted upper limit value.

(4) A Posteriori Distribution at Time $t+1$

$$
\begin{aligned}
\left(\boldsymbol{\mu}_{t+1} \mid \mathbf{D}_{t+1}\right) & =\left(\left[\begin{array}{c}
\theta_{t+1} \\
\Delta_{t+1} \\
\xi_{t+1}+6 a
\end{array}\right] \mid \mathbf{D}_{t+1}\right) \\
& \sim N\left[\mathbf{m}_{t+1}, \mathbf{C}_{t+1}\right],
\end{aligned}
$$

where, $\mathbf{A}_{t+1}=\mathbf{R}_{t+1} Q_{t+1}^{-1}, e_{t+1}=y_{t+1}-f_{t+1}, \mathbf{m}_{t+1}=\mathbf{a}_{t+1}+$ $\mathbf{A}_{t+1} e_{t+1}, \mathbf{C}_{t+1}=\mathbf{R}_{t+1}-\mathbf{A}_{t+1} Q_{t+1} \mathbf{A}_{t+1}^{\prime}$.

\section{Monitoring Mechanisms about BDLM}

Model monitoring has three purposes. The first is to identify where model prediction function declines and in which form the model fault occurred. The second is to cope with the faults and to monitor and update the model. The third is to improve the accuracy of future prediction.

In this paper, the main idea of monitoring mechanism is to construct Bayesian factors $[5,7,8,11-14]$ with the obtained one-step prediction distribution function of the built BDLM and the routine or standard probability model, where Bayesian factors include single one and cumulative one.

The single Bayesian factor for $p_{0}(\cdot)$ versus $p_{1}(\cdot)$ based on the monitored data $y_{t}$ is defined as

$$
H(t+1)=\frac{p_{0}\left(y_{t+1} \mid \mathbf{D}_{t}\right)}{p_{1}\left(y_{t+1} \mid \mathbf{D}_{t}\right)}
$$

where $p_{0}\left(y_{t+1} \mid \mathbf{D}_{t}\right)$ is the one-step predictive PDF about monitored extreme stress. $p_{1}\left(y_{t+1} \mid \mathbf{D}_{t}\right)$ is the PDF of the alternative model, namely, the routine or standard PDF. $H(t+$ $1)$ is the Bayesian factor for $p_{0}\left(y_{t+1} \mid \mathbf{D}_{t}\right)$ versus $p_{1}\left(y_{t+1} \mid \mathbf{D}_{t}\right)$ based on the monitored data $y_{t+1}$.

For integers $k=1, \ldots, t$, the Bayesian factor for $p_{0}(\cdot)$ versus $p_{1}(\cdot)$ based on the sequence of $k$ consecutive monitored data $y_{t+1}, y_{t}, \ldots, y_{t-k+2}$ is built as (23), namely, the built formula $H_{t+1}(k)$ of the cumulative Bayesian factor is

$$
\begin{aligned}
H_{t+1}(k) & =\prod_{r=t-k+2}^{t+1} H_{r} \\
& =\frac{p_{0}\left(y_{t+1}, y_{t}, \ldots, y_{t-k+2} \mid \mathbf{D}_{t-k+1}\right)}{p_{1}\left(y_{t+1}, y_{t}, \ldots, y_{t-k+2} \mid \mathbf{D}_{t-k+1}\right)},
\end{aligned}
$$

where $H_{t+1}(k)$ is the cumulative Bayesian factor, which measures the evidence provided by the recent (up to and including time $t+1) k$ consecutive monitored data $y_{t+1}, y_{t}, \ldots, y_{t-k+2}$.

Further through simplifying (22), a useful single alternative is the scale shift model $p_{1}(\cdot)$ in which $e_{\text {te }}$ has standard deviation $k$ rather than unity, with

$$
p_{1}\left(e_{\mathrm{te}} \mid \mathbf{D}_{t}\right)=\left(2 \pi k^{2}\right)^{-0.5} \exp \left\{-0.5\left(\frac{e_{\mathrm{te}}}{k}\right)^{2}\right\} \text {. }
$$

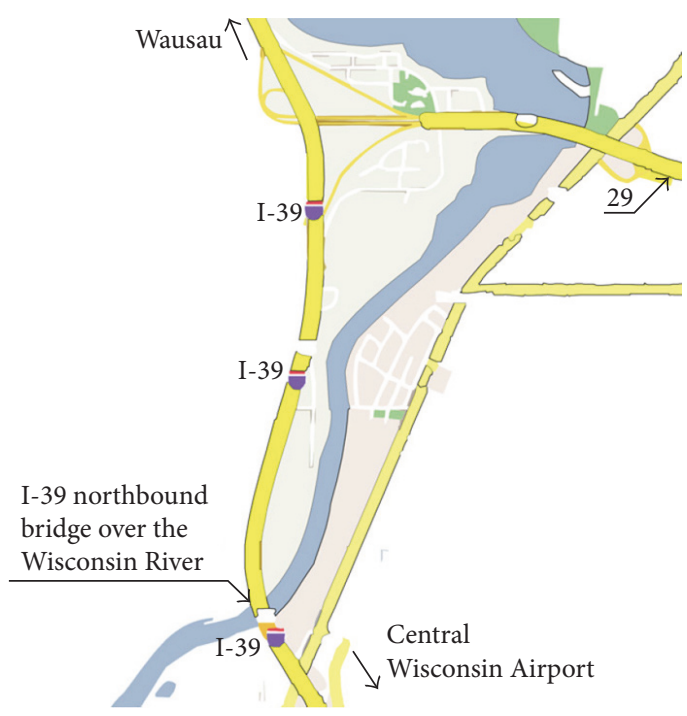

FIGURE 3: Map view of the I-39 northbound bridge (adapted from [9]).

Then the specific expression of the Bayesian factor is obtained as

$$
H(t)=k \exp \left\{-0.5 e_{\text {te }}^{2}\left(1-k^{-2}\right)\right\},
$$

where $e_{\mathrm{te}}=\left(y_{t+1}-f_{t+1}\right) / Q_{t+1}^{0.5}$ is the standardized prediction errors.

Monitoring rules of the Bayesian dynamic model is when $k=3$, if $H(t+1)$ is less than 0.15 ; then the corresponding monitored data is abnormal, which need to be deleted. And after the removal of the abnormal data, with the changing curves of cumulative Bayesian factor, the prediction precision of the Bayesian dynamic model can be reflected. Namely, if the cumulative Bayesian factor is bigger, the prediction precision of the BDLM is better, and the uncertainty of the BDLM is smaller.

\section{Application to an Existing Bridge}

The I-39 northbound bridge $[5,9]$ shown in Figure 3 was built in 1961. The extreme stresses of the sensor $\mathrm{CH} 15$ is monitored for eighty-two days, and this sensor $\mathrm{CH} 15$ was mounted on the bottom flange of the northbound bridge girder (see Figure 4). The strain gage was installed in span two (first lateral field) at P.P.9 on the girder G4, as shown in Figure 4 . The sensor was located at this position, since the stress concentrations and crack initiations due to the welded flange cover plates associated with field splices are significant at that detail [9].

The monitored data of the sensor $\mathrm{CH} 15$ displayed the variability of the stresses caused by traffic, temperature, shrinkage, creep, and structural changes. The stresses from the dead weight of the steel structure and the concrete deck are not included in the measured data. The monitored extreme stresses are shown in Table 1.

Now, the monitored extreme stress data of the forward seventy days is adopted to predict the extreme stress of 

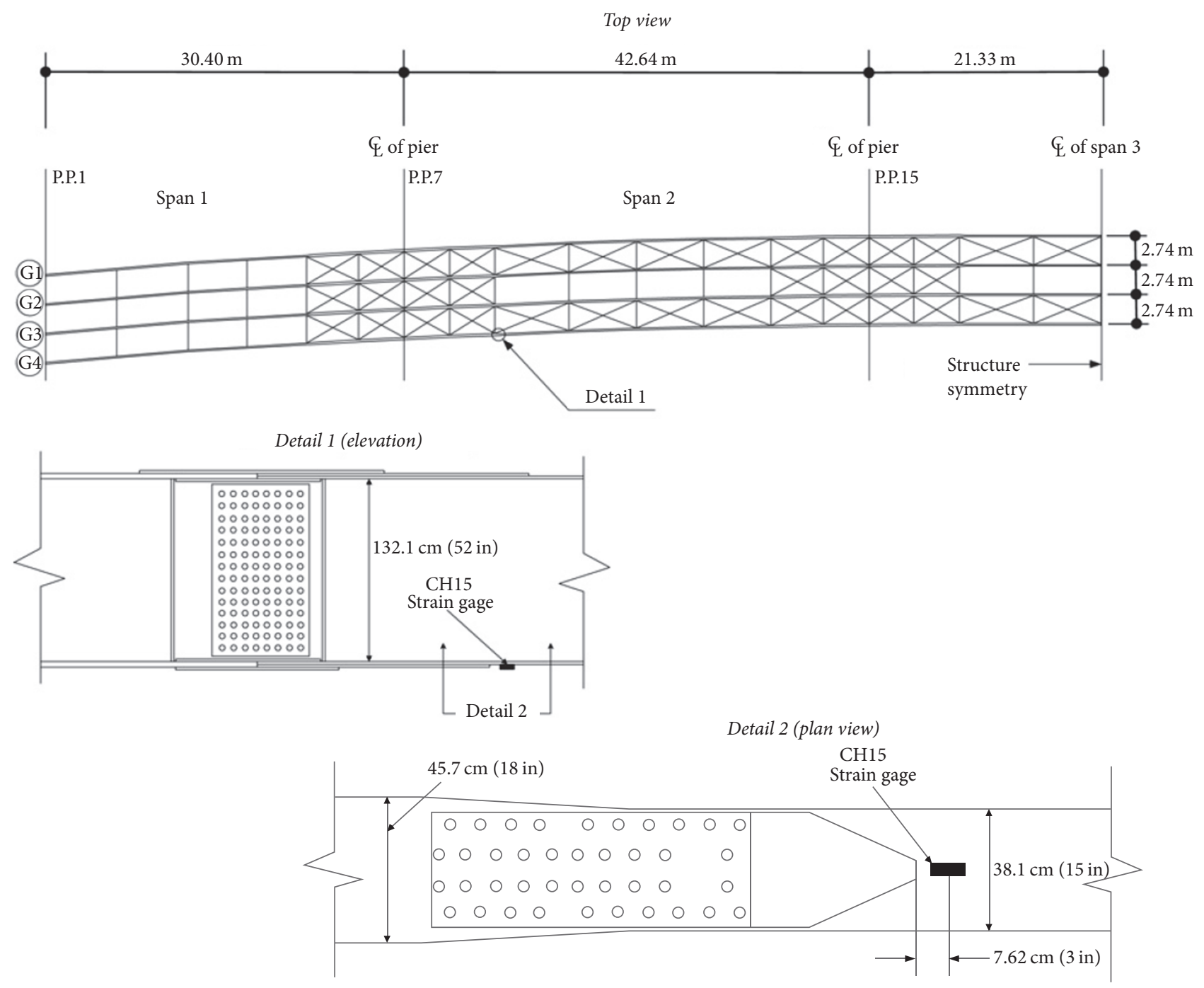

FIGURE 4: I-39 northbound bridge, instrumentation plan of the strain gage, CH15 (adapted from [9]).

the later twelve days based on the built BDLM shown in (27)-(29). The detailed building steps are described as follows.

In this existing example, the state data, which is shown in Figure 5, is obtained with the resampled method, and the state equations obtained with (1)-(4) are adopted to build the BDLM and predict the structural extreme stress. From Figure 6 and Table 2, it can be seen that the RMSE about the cubic polynomial function is lowest. So (1) is used to fit the trend data of monitored data, namely,

$$
m_{t}=-0.0001245 t^{3}+0.01246 t^{2}-0.2956 t+24.06
$$

where $m_{t}$ is approximately state value of monitored data at time $t, a=-0.0001245, b=0.01246, c=-0.2956$, and $d=24.06$. Equation (26) can be used to build state equations shown in (28).

In order to solve the distribution parameters of initial state information, the monitored extreme stress data of the forward seventy days is resampled with cubical smoothing algorithm with five-point approximation; then the resampled data $\theta_{t}$ and the responding differences $\left(\Delta_{t}, \xi_{t}\right)$ are obtained, which can be approximately considered as the initial state information and, respectively, shown in Figure 7.

Through Kolmogorov-Smirnov (K-S) test for the initial state data and initial state difference data, with (12), the optimum a priori PDF of initial state is normal PDF shown in (29).

With (5)-(8), the built BDLM is as follows.

Monitored equation is

$$
y_{t+1}=\theta_{t+1}+v_{t+1}, \quad v_{t+1} \sim N\left[0, V_{t+1}\right] \text {. }
$$

State equation is

$$
\left(\begin{array}{c}
\theta_{t+1} \\
\Delta_{t+1} \\
\xi_{t+1}
\end{array}\right)=\mathbf{G}_{t+1}\left(\begin{array}{c}
\theta_{t} \\
\Delta_{t} \\
\xi_{t}+6 a
\end{array}\right)+\omega_{t+1},
$$


TABLE 1: Dynamic monitored extreme stresses $[9,14]$.

\begin{tabular}{|c|c|}
\hline Time (day) & Stress $\sigma(\mathrm{MPa})$ \\
\hline 1 & 25.23 \\
\hline 2 & 21.67 \\
\hline 3 & 19.53 \\
\hline 4 & 20.50 \\
\hline 5 & 24.44 \\
\hline 6 & 22.66 \\
\hline 7 & 25.95 \\
\hline 8 & 27.65 \\
\hline 9 & 25.26 \\
\hline 10 & 21.40 \\
\hline 11 & 21.48 \\
\hline 12 & 20.06 \\
\hline 13 & 20.60 \\
\hline 14 & 22.56 \\
\hline 15 & 23.54 \\
\hline 16 & 20.94 \\
\hline 17 & 19.16 \\
\hline 18 & 22.47 \\
\hline 19 & 23.37 \\
\hline 20 & 20.99 \\
\hline 21 & 24.15 \\
\hline 22 & 21.22 \\
\hline 23 & 22.02 \\
\hline 24 & 24.80 \\
\hline 25 & 22.51 \\
\hline 26 & 21.57 \\
\hline 27 & 21.67 \\
\hline 28 & 19.16 \\
\hline 29 & 21.67 \\
\hline 30 & 23.99 \\
\hline 31 & 21.05 \\
\hline 32 & 23.35 \\
\hline 33 & 22.66 \\
\hline 34 & 24.61 \\
\hline 35 & 25.77 \\
\hline 36 & 26.54 \\
\hline 37 & 22.83 \\
\hline 38 & 21.05 \\
\hline 39 & 24.44 \\
\hline 40 & 28.80 \\
\hline 41 & 20.24 \\
\hline 42 & 29.97 \\
\hline 43 & 24.17 \\
\hline 44 & 23.72 \\
\hline 45 & 26.85 \\
\hline 46 & 25.32 \\
\hline 47 & 26.93 \\
\hline 48 & 25.06 \\
\hline 49 & 23.01 \\
\hline
\end{tabular}

TABLE 1: Continued.

\begin{tabular}{|c|c|}
\hline Time (day) & Stress $\sigma(\mathrm{MPa})$ \\
\hline 50 & 22.02 \\
\hline 51 & 23.90 \\
\hline 52 & 20.10 \\
\hline 53 & 25.24 \\
\hline 54 & 25.77 \\
\hline 55 & 27.11 \\
\hline 56 & 23.72 \\
\hline 57 & 22.65 \\
\hline 58 & 24.89 \\
\hline 59 & 27.56 \\
\hline 60 & 25.86 \\
\hline 61 & 24.61 \\
\hline 62 & 22.11 \\
\hline 63 & 21.22 \\
\hline 64 & 25.15 \\
\hline 65 & 24.64 \\
\hline 66 & 23.18 \\
\hline 67 & 21.94 \\
\hline 68 & 21.82 \\
\hline 69 & 22.66 \\
\hline 70 & 21.57 \\
\hline 71 & 29.16 \\
\hline 72 & 21.57 \\
\hline 73 & 22.92 \\
\hline 74 & 21.94 \\
\hline 75 & 21.14 \\
\hline 76 & 20.41 \\
\hline 77 & 22.76 \\
\hline 78 & 22.38 \\
\hline 79 & 25.21 \\
\hline 80 & 24.98 \\
\hline 81 & 23.82 \\
\hline 82 & 26.44 \\
\hline
\end{tabular}

TABLE 2: RMSE about the regressive polynomial functions.

\begin{tabular}{lc}
\hline Functions & RMSE \\
\hline Linear polynomial & 2.305 \\
Quadratic polynomial & 2.303 \\
Cubic polynomial & 2.167 \\
\hline Note. RMSE: root-mean-square error. &
\end{tabular}




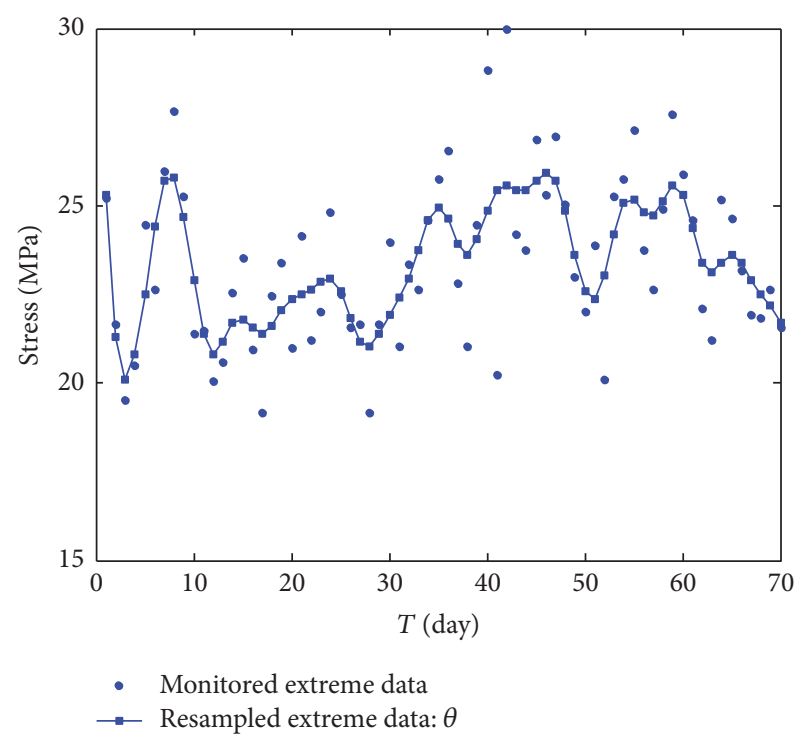

Figure 5: Monitored extreme stress data and resampled extreme stress data.

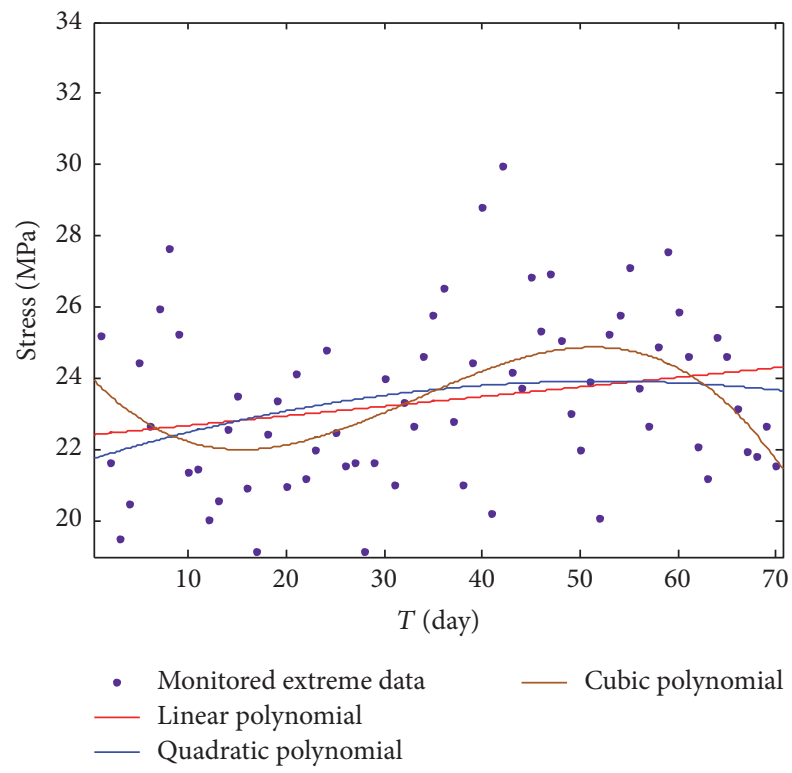

FIGURE 6: Monitored data and its regression analysis.

Initial state information is

$$
\left(\left[\begin{array}{c}
\theta_{t} \\
\Delta_{t} \\
\xi_{t}+6 a
\end{array}\right] \mid \mathbf{D}_{t}\right) \sim N\left[\mathbf{m}_{t}, \mathbf{C}_{t}\right]
$$

where $V_{t+1}=V=2.4344$ estimated with the monitored extreme stress data of the forward seventy days.

$$
\mathbf{m}_{t}=\left[\begin{array}{c}
m_{\theta_{t}} \\
m_{\Delta_{t}} \\
m_{\xi_{t}}
\end{array}\right]=\left[\begin{array}{c}
23.3750 \\
-0.0519 \\
-0.0078
\end{array}\right],
$$

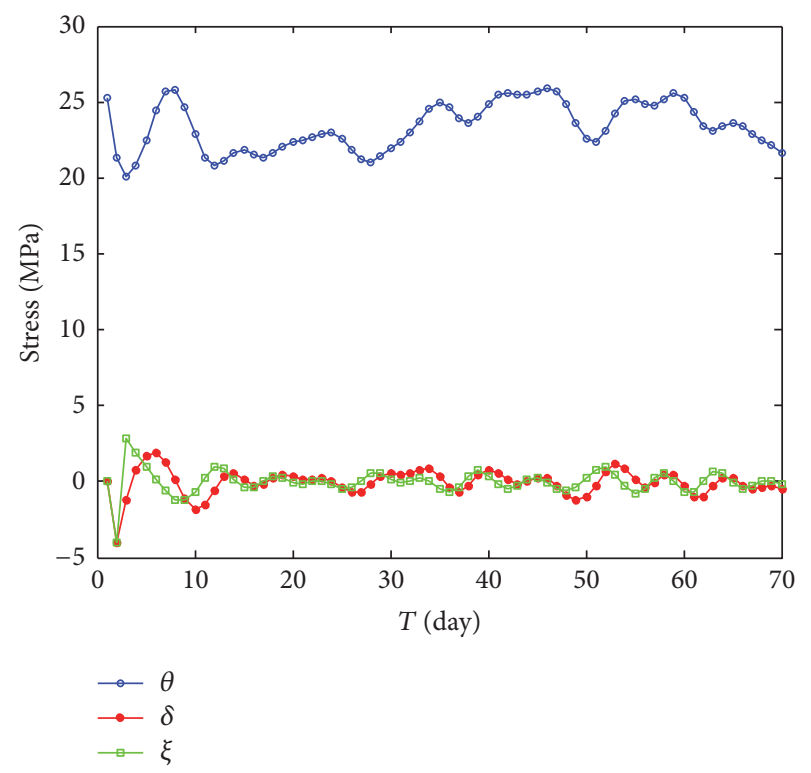

FIGURE 7: Initial state data and initial state difference data.

$$
\begin{aligned}
\mathbf{G}_{t+1}= & \left(\begin{array}{ccc}
1 & 1 & \frac{r_{i+1}}{r_{i}} \\
0 & 1 & \frac{r_{i+1}}{r_{i}} \\
0 & 0 & \frac{r_{i+1}}{r_{i}}
\end{array}\right)=\left(\begin{array}{lll}
1 & 1 & 1 \\
0 & 1 & 1 \\
0 & 0 & 1
\end{array}\right), \\
\mathbf{C}_{t}= & {\left[\begin{array}{ccc}
C_{\theta_{t}} & C_{(\theta, \Delta)_{t}} & C_{(\theta, \xi)_{t}} \\
C_{(\theta, \Delta)_{t}} & C_{\Delta_{t}} & C_{(\Delta, \xi)_{t}} \\
C_{(\xi, \theta)_{t}} & C_{(\xi, \Delta)_{t}} & C_{\xi_{t}}
\end{array}\right] } \\
= & {\left[\begin{array}{ccc}
2.5043 & 0.3506 & -0.3820 \\
0.3506 & 0.7124 & 0.3206 \\
-0.3820 & 0.3206 & 0.6383
\end{array}\right], } \\
W_{t+1}= & \left(\begin{array}{ccc}
\frac{1-\delta}{\delta} & G_{t+1} C_{t} G_{t+1}^{\prime} \\
= & 0 & 0 \\
0 & 0.0145 & 0 \\
0 & 0 & 0.0130
\end{array}\right],
\end{aligned}
$$

where $y_{t+1}$ is the monitored extreme stress data at time $t+1$. $v_{t+1}$ is monitored error. $\boldsymbol{\omega}_{t+1}$ is state error vector, which means the uncertainty of state variable. $\delta=0.98, N[\cdot]$ is normal PDF.

With (17)-(21) and (27)-(29), the predicted extreme stresses and prediction precision (the reciprocal of prediction variances) can be solved and, respectively, shown in Figures 8 and 9.

In Figure 8, it is noticed that the predicted stress data and the predicted stress ranges fit the changing rules of monitored extreme data. And Figure 9 shows that the prediction precision of the BDLM increases with the updating of the distant monitored data. 


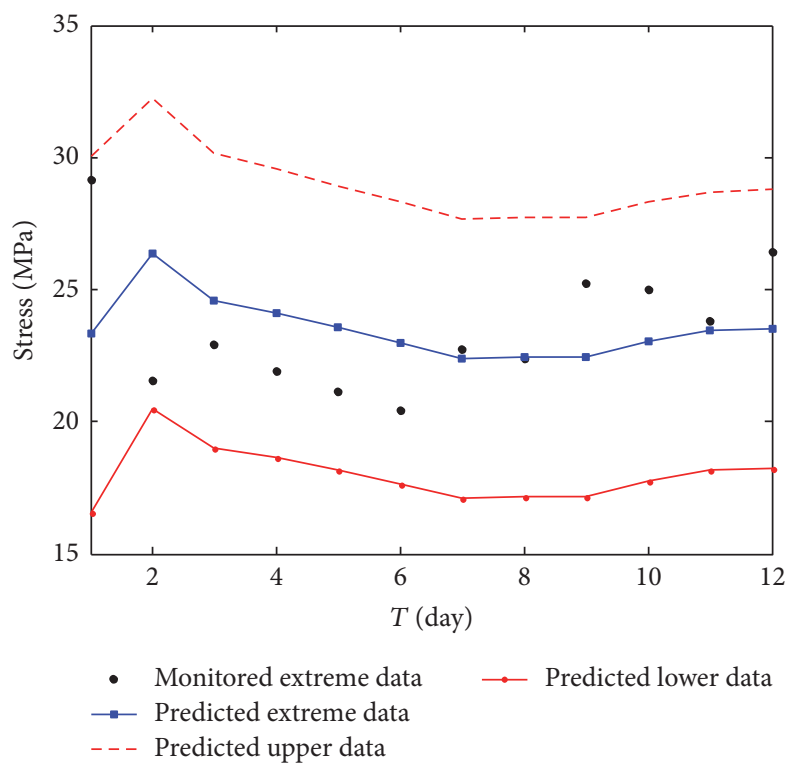

FIGURE 8: Monitored and predicted extreme stress data.

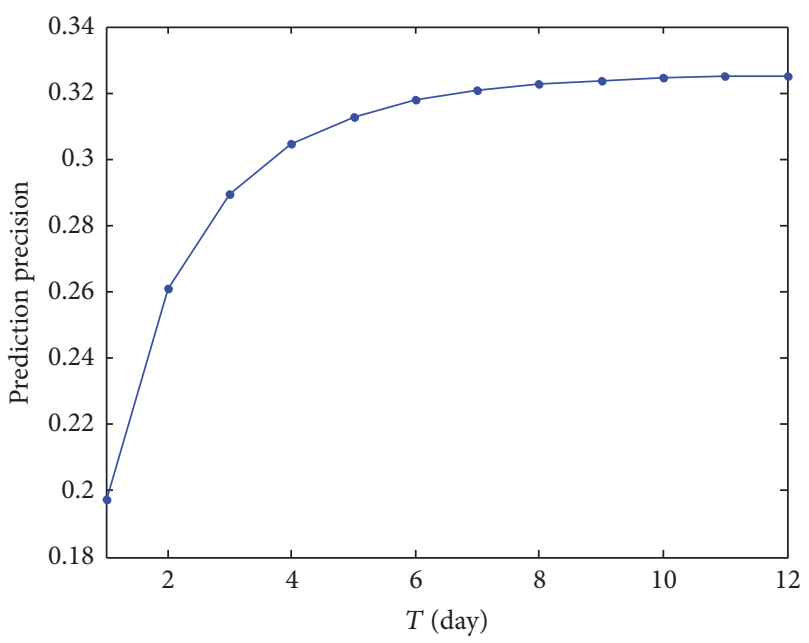

FIGURE 9: Prediction precision of BDLM.

Based on (22)-(25) and the built BDLM, the model monitoring results are figured in Figures 10 and 11. Figure 10 shows that Bayesian factors are all bigger than 0.15 , which mean that all the monitoring data is normal. Figure 11 shows that the cumulative Bayesian factors approximately increase with the distant updating of monitored data, which mean that the prediction precision of the BDLM is better and better, and the corresponding model uncertainty is smaller and smaller.

\section{Conclusions}

The paper describes a new approach for incorporating monitored data into structural extreme stress prediction. Namely, based on the monitored extreme stress data including nonlinear effects, the cubic function-based BDLM is utilized in this

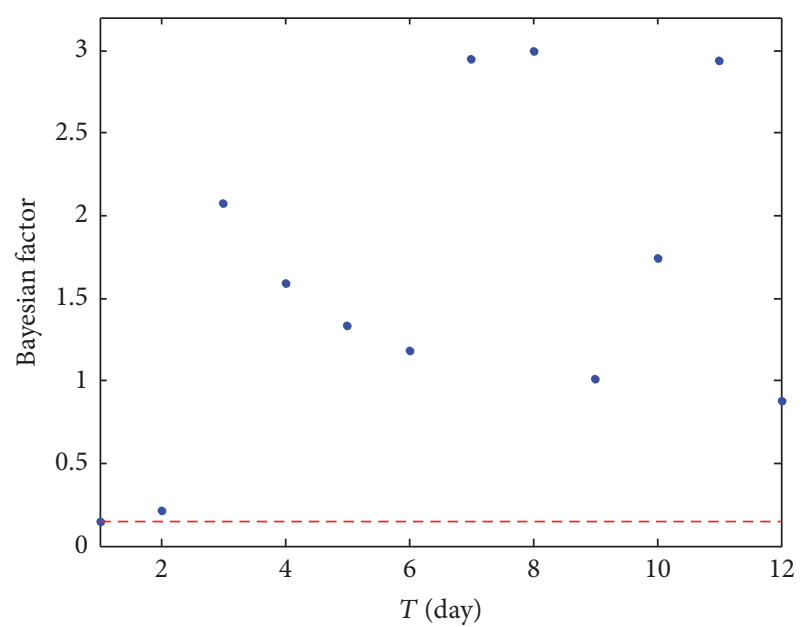

- Single data

- - - Threshold value: 0.15

FIGURE 10: Single Bayesian factors of BDLM.

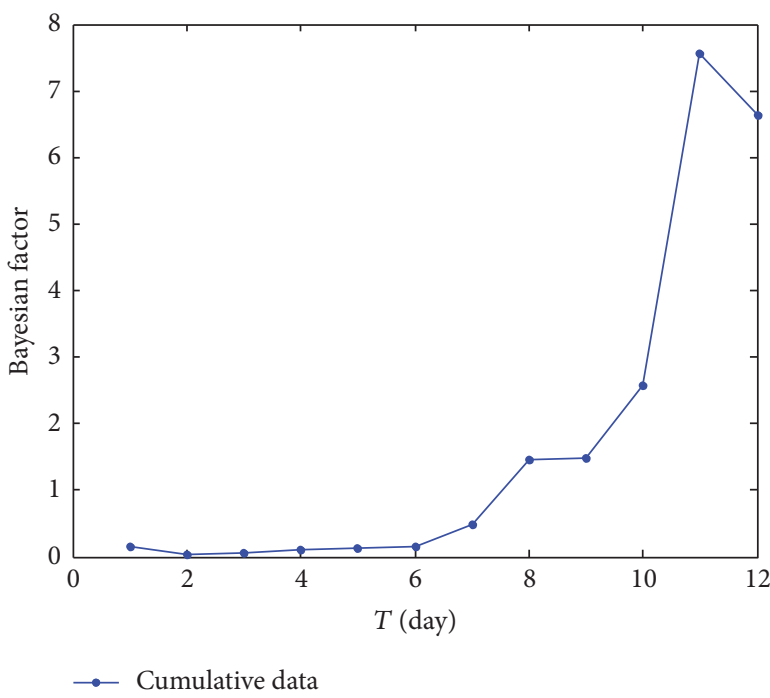

FIGURE 11: Cumulative Bayesian factors of BDLM.

paper. This kind of model is easy to build and can be widely used in the bridge performance prediction.

\section{Conflicts of Interest}

The authors declared no potential conflicts of interest with respect to the research, authorship, and/or publication of this article.

\section{Acknowledgments}

This work was supported by the National Natural Science Foundation of China (Project nos. 51608243 and 51702142), the Natural Science Foundation of Gansu Province of China (Project no. 1606RJYA246), the Fundamental Research Funds for the Central Universities (Project nos. lzujbky-2015-300, 
lzujbky-2015-301, and lzujbky-2017-k17) for the research, authorship, and/or publication of this article.

\section{References}

[1] Li. SL, Approaches of Condition Assessment and Damage Alarming of Bridges based on Structural Health Monitoring, School of Civil Engineering, Harbin Institute of Technology, Harbin, China, 2009, (in Chinese).

[2] S. S. Law and J. Li, "Updating the reliability of a concrete bridge structure based on condition assessment with uncertainties," Engineering Structures, vol. 32, no. 1, pp. 286-296, 2010.

[3] J. Li and H. Hao, "Damage detection of shear connectors under moving loads with relative displacement measurements," Mechanical Systems and Signal Processing, vol. 60, pp. 124-150, 2015.

[4] J. Li, H. Hao, and J. V. Lo, "Structural damage identification with power spectral density transmissibility: numerical and experimental studies," Smart Structures \& Systems, vol. 15, no. 1, pp. 15-40, 2015.

[5] X. P. Fan, Reliability updating and bayedsian prediction of bridges based on proof loads and monitoring data [Ph.D. thesis], Harbin Institute of Technology, Harbin, China, 2014.

[6] X. P. Fan and D. G. Lu, "Real-time reliability forecast of bridge structures based on multiple Bayesian dynamic linear models," Journal of South China University of Technology, vol. 41, no. 3, pp. 67-72, 2013, (in Chinese).

[7] X. P. Fan and D. G. Lu, "Reliability prediction of bridges based on monitored data and Bayesian dynamic models," Key Engineering Materials, vol. 574, pp. 77-84, 2014.

[8] X. P. Fan and D. G. Lu, "Reliability prediction of aging bridges based on Bayesian dynamic linear model," Journal of the china railway society, vol. 36, no. 6, pp. 93-98, 2014 (Chinese).

[9] D. M. Frangopol, A. Strauss, and S. Kim, "Use of monitoring extreme data for the performance prediction of structures: General approach," Engineering Structures, vol. 30, pp. 36443653, 2008.

[10] X. P. Fan and Lu D. G., "Reliability prediction of bridge structures based on BDNM," Journal of Harbin Institute of Technology, vol. 46, no. 2, pp. 1-5, 2014.

[11] M. West and J. Harrison, Bayesian forecasting and dynamic models, Springer series, New York, 2nd edition, 1997.

[12] G. Petris, S. Petrone, and P. Campagnoli, Dynamic linear models with $R$, Springer series, New York, 2009.

[13] J. Wang and X. L. Liu, "Evaluation and Bayesian dynamic prediction of deterioration of structural performance," Structure and Infrastructure Engineering: Maintenance, Management, Life-Cycle Design and Performance, vol. 6, no. 6, pp. 663-674, 2010.

[14] J. Wang, Performance prediction and reliability management of concrete structures based on information updating, School of Civil Engineering, Tsinghua University, Beijing, China, 2006, (in Chinese). 

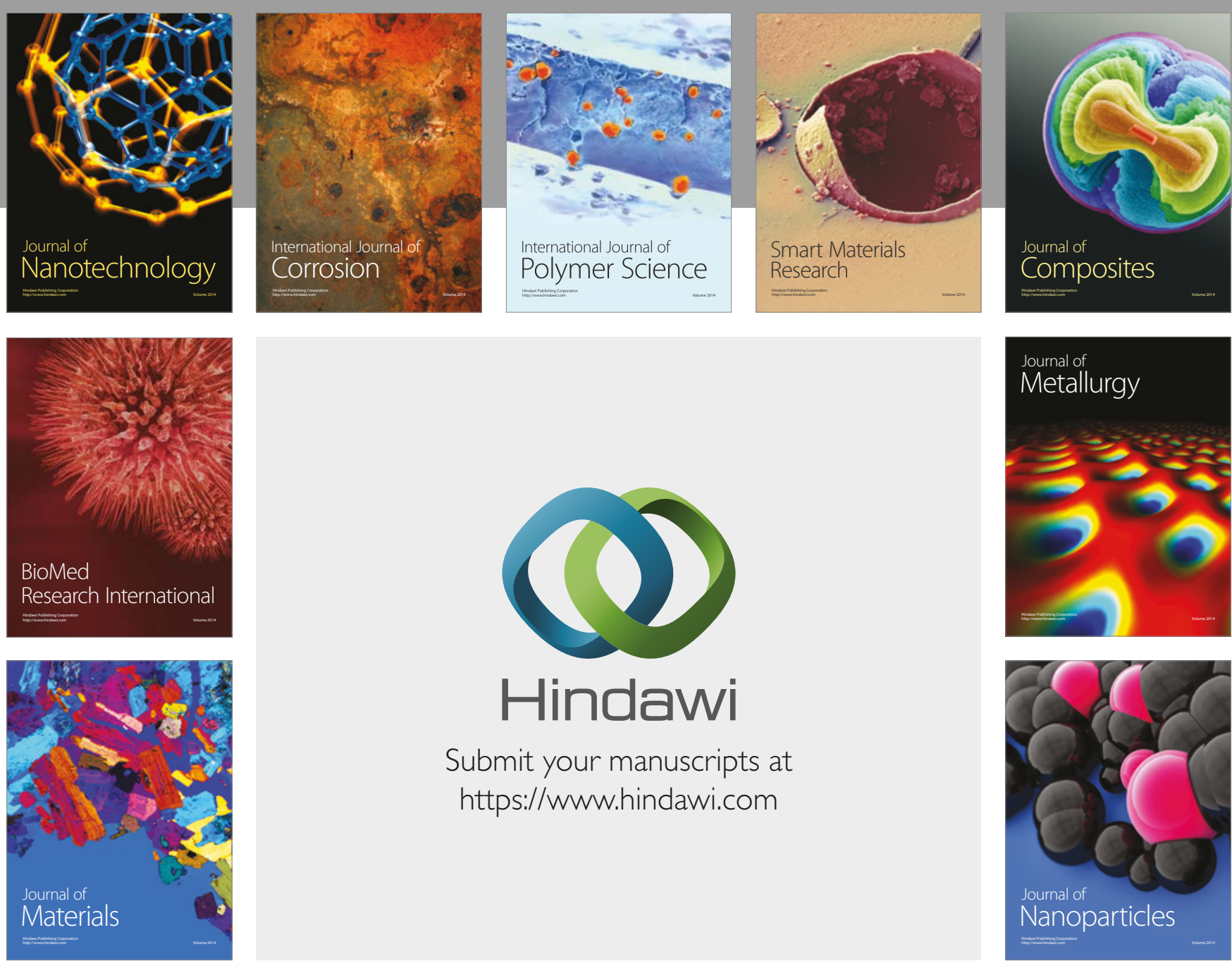

\section{Hindawi}

Submit your manuscripts at

https://www.hindawi.com
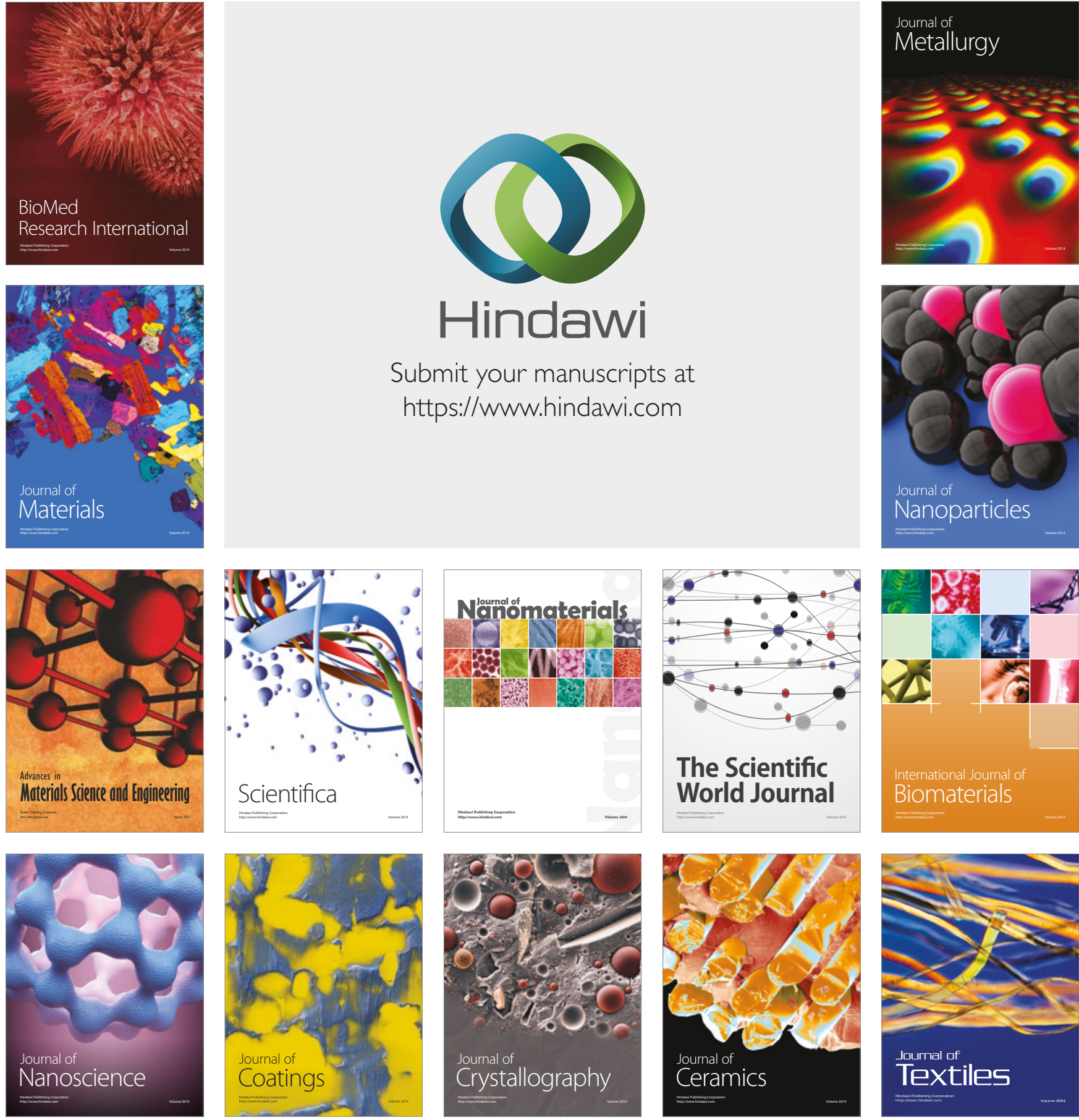

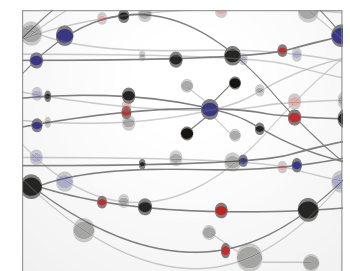

The Scientific World Journal
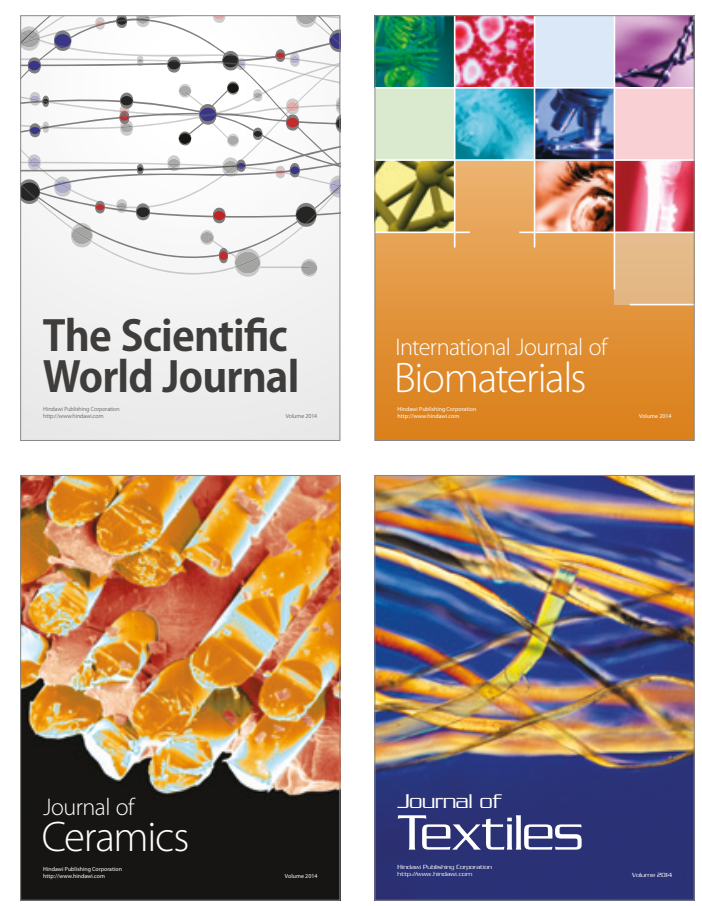